\title{
An analysis of mobility management for mobile sensor nodes in MWSN
}

\author{
Zhang Min, Jun-Bin Liang \\ France Guangxi Key Laboratory of Multimedia Communications and Network Technology, School of Computer and Electronics \\ Information, Guangxi University, Nanning 530004, China
}

\begin{abstract}
Mobile wireless sensor networks (MWSN) are composed of a large number of mobile sensor nodes, which are used to collect data. MWSN have been widely applied in a variety of harsh environments, so mobile sensor nodes are often at risk of damage. How to manage mobile sensor nodes is an important issue. In this paper, we analyse different mobility management schemes proposed in some typical research literature and classify these schemes by using three features: the collaboration between mobile sensor nodes, routing and deployment in MWSN. We specify advantages and disadvantages of the proposed schemes, compare different key parameters of MSWN respectively, including energy efficiency, data delay, cost and lifetime, etc. Finally, we discuss existing problems of MWSN management and give some helpful suggestions in this area.
\end{abstract}

\section{Introduction}

In recent years, Mobile wireless sensor networks (MWSN) have been widely applied in a variety of harsh environments, such as coastal and border protection, search and rescue, battlefield reconnaissance, underwater surveillance, air traffic control, target tracking. MWSN are composed of a large number of distributed sensor nodes, these nodes organize themselves into multi-hop wireless networks, and each node is capable of performing computational operations, storage and communication functions [11]. The sensor device is battery powered, it is not feasible to replace the node battery in a large-scale deployment, therefore energy is the most valuable resource in MWSN. Mobile sensor nodes are often at risk of damage, such as one node fails in the network, corrupts and adds a new node, or causes the sensor node to exhaust its energy for some reasons, then the sensor network changes its topology. Therefore, we need to pay attention to the mobility management of sensor nodes in MWSN urgently.

In MWSN, there are various literature provide some more flexible, powerful and reliable schemes by fully addressing issues related to self-organization of networks and network coverage and lifetime. However, these schemes add the complexity of the mobility management algorithms in terms of cost[1].

In this paper, we focus on mobility management features for sensor nodes: deployment, routing, collaboration of mobile nodes. we classify the MWSN mobility management schemes (Figure 1).

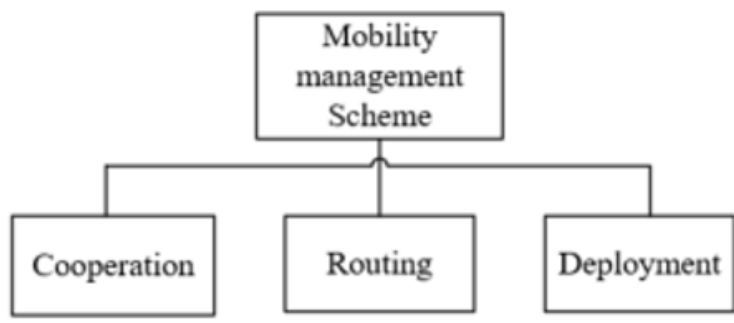

Figure 1. Classification of Mobility management

In section 2, we describe the typical mobility management schemes. In section 3, we compare the performance of these schemes in detail. In section 4 we provide some suggestions for the future research work. Finally, we summarize our work in section 5.

\section{Management schemes for sensor nodes}

At present, a large number of researchers have proposed mobility-related schemes for sensor nodes in MWSN, which have significant advantages in saving energy, improving scalability and extending network life.

\subsection{Cooperation}

Mobile nodes cooperate with static nodes could offer important benefits in all aspects of MWSN. We analyse the collaboration between mobile sensor nodes through the follow Random waypoint model and Routing based Nodes Collaboration under Random WayPoint method. 


\subsubsection{Random waypoint model}

In the first research, Johnson and Maltz proposed the Random WayPoint (RWP)model to evaluate the dynamic routing protocol DSR, which was subsequently improved [8]. Due to the simplicity and wide availability of the RWP model, it plays an important role in mobility management. In mobility management, the mobile node is randomly distributed in the simulated area, we need to simulate all scenarios that a node moves toward a destination, pause time at arrival, then move to another destination, and so on. In this model, each node is randomly selected as the target point of the coordinates ( $\mathrm{x}$, $\mathrm{y})$ in the simulation surface, and the velocity between 0 and Vmax. The node trips to the selected destination with the selected speed. Upon arrival, the node selects a new destination and a new speed again before the pause time to repeat the same process.

\subsubsection{Routing based Nodes Collaboration under Random WayPoint}

Lyamineet et al. [2] designed a new routing method RNC-RWP (Routing based Nodes Collaboration under Random WayPoint) which is able to route information in a mobile environment. The node of the RNC-RWP model is free to move in the environment (according to the model RWP). During its pause time, each Y node updates its one hop neighbor table (Vy) and will follow the prescribed steps. First, we assume that there are no obstacles in the environment throughout the simulation, then use the TOSSIM simulator for the experimental evaluation. In terms of performance analysis, RNC-RWP has a better transmission rate and lower data latency than RWP. The results show that RNC-RWP provides a huge benefit in the end-to-end latency, which is the result of the collaboration between mobile sensor nodes.

\subsection{Deployment}

The deployment of mobile sensor nodes influences the network connectivity and lifetime of the sensors. We study the connectivity by comparing the OBA model, the CPANMCR method and OACRPANM method, survey various node mobility Scenarios based on B-MAC Protocol.

\subsubsection{Network connectivity}

Mobile Wireless Sensor Network has been widely used in hash environments. Since the limited airborne energy, sensor nodes can easily fail in hashed environments, which can cause the network to split into disjoint islands.

\subsubsection{OBA}

Since mobility consume away a great deal of energy and can be limited by terrain constraints, the moving distance of mobile sensor nodes should be minimized in the recovery work. In [9], Sir, Mustafa Y., et al. proposed a mathematical model (OBA)that minimizes the total travel distance connecting a given number of partitions. Based on network flow, the problem is modeled as a mixed integer nonlinear program. Nonlinear terms in the model are linearized with a polygonal approximation to improve computational efficiency. Compared with the heuristic approach, the method is better in total distance and delay,and reveals the various trade-offs involved in connecting multiple partitions.

\subsubsection{CPANMCR}

In [10], Chun-hui WU, et al. proposed a novel method, CPANMCR, which combines power adjustment and node movement (CPANMCR) to restore network connectivity. There are three main stages in CPANMCR: firstly detect the boundary node of each island, then adjust the power of the boundary node to restore the connection, and finally move the mobile node to the appropriate location to restore the connectivity of the entire network. A large number of simulation experiments show that this method can ensure network connectivity and reduce the total travel distance.

\subsubsection{OACRPANM}

Chun-hui WU, et al. proposed a new method OACRPANM in ]3], which avoid obstacle to restore the networks based on power adjustment and node's movement (OACRPANM). First, the boundary nodes of each island are detected, and then the transmission power of the boundary nodes is adjusted to restore network connectivity. If adjusting the power does not restore the connectivity of the entire network, performing a third phase involving moving the mobile node avoiding the obstacle to the appropriate location to recover the entire network. In the simulation experiment, Chun-hui WU, et al mainly consider the total distance and the number of mobile nodes and islands. The results show that the total distance decreases as the mobile node increases, and the total distance of OACRPANM or CPANMCR is always lower than the OBA, this indicates that the proposed OACRPANM is more effective than OBA. Since the obstacles in the moving path are considered in ]3], the total distance of the OACRPANM algorithm is always higher than CPANMCR. However, OACRPANM is more effective in the actual environment, which also shows that the OACRPANM algorithm saves more energy costs and is more efficient when the number of mobile nodes increases.

\subsection{2 lifetime of the sensors}

In MWSN, mobile sensor nodes are battery powered, which is a challenging problem when deploying a network. The main goal is to reduce battery consumption to allow these sensors to extend their lifetime. In order to extend the lifetime of the sensors, it must be ensured that the deployment of such nodes is effective in terms of energy consumption in some cases. Three scenarios is mentioned: fixed or non-mobility scenes, linear motion scenes, circular capacity scenes, the simulation is based 
on B-MAC protocol implemented on WSNs under different mobility framework modules [4]. The simulation results show that the performance of the BMAC protocol can vary according to energy consumption under different conditions. The purpose is to generate results based on different scenarios regarding mobility and check WSN performance in terms of total battery power consumed. The results are used for sensor deployment to extend sensor node operating time. The energy consumption in a node depends on two factors discussed, mobility and energy. The conclusion is that a static scenario is best to save energy over a longer period of time. Fixed mode is energy efficient since it does not involve other issues such as mobility, speed, rotation, it helps the node to focus on the calculations needed, so the only energy consumed is the calculations for the nodes.

\subsection{Routing}

The routing's relevant issues including network structure based, mobility based on mobile sensors or relays. We study the routing of MWSN through a distributed ResAll algorithm and The FRAVD Communication scheme.

\subsubsection{ResAll algorithm}

In MWSN, scavenging energy from ambient radio frequency (RF) signals is a promising solution to prolonging the lifetime of energy-constrained relay nodes. In the literature ]5], Guo, Songtao, et al. apply simultaneous Wireless Information and Power Transfer (SWIPT) technique to the MWSN, where the energy collected by the relay node can compensate for its energy consumption on data forwarding [7]. By combining the receiver's circuit power consumption and RF energy harvesting capabilities into the objective function, the resource allocation problem is expressed as a non-convex optimization problem, the minimum individual and system data rate requirements of the receiver, minimum power transfer and maximum system power consumption are considered. In order to solve the convex problem, Guo, Songtao, et al. propose a distributed ResAll algorithm, through the algorithm, the transmitter adaptively adjusts its optimal data rate and allocates its optimal transmit power, while the receiver dynamically adjusts its optimal power allocation ratio to maximize system energy efficiency. The simulation results show that the algorithm converges in a small number of iterations, which can effectively supplement the energy of the sensor nodes and improve energy efficiency.

\subsubsection{The FRAVD Communication scheme}

In a mobile wireless sensor network control system, mobile nodes constantly move to detect events, while static nodes constitute a communication infrastructure for information transmission. Therefore, how to communicate with the receiver quickly and efficiently is an important research topic of the MWSN control system.
In [6], authors propose a communication scheme named first relay node selection based on fast response and multi-hop relay transmission with variable duty cycle (FRAVD). This scheme can effectively reduce network delay by combining the first relay node selection with the node duty cycle setting. In the FRAVD, for the first relay node selection, authors propose a fast response-based strategy that selects the first relay node from neighboring nodes within the communication range in the shortest response time and guarantees the remaining energy. The receiving distance with the node is better than the average. Then for the multi-hop data transmission of static nodes, the novelty of variable duty cycle is introduced, and the residual energy is used to improve the duty ratio of the nodes in the far-end region. Because the node uses sleepwake asynchronous mode, it can significantly increase the duty cycle to improve network performance in terms of latency and transmission reliability.

The comprehensive performance analysis shows that compared with the communication scheme with fixed duty cycle, the FRAVD scheme has a lower network delay and increases the probability of discovering the first relay node to a certain degree, while also ensuring that the network life cycle is higher than previous studies, it is a comparatively effective low-latency communication scheme.

\section{Comparison}

According to the classification of mobile management schemes, a comparison (Table 1) is performed in terms of energy efficiency, data delay, cost, lifetime, simulation /Experimental test platform used, the type of simulator used.

From Table 1, we can draw conclusions: energy efficiency is the primary indicator considered by all mobility management schemes. Data latency issues are also considered when focusing on collaboration between mobile nodes. When focusing on mobile node deployment, the solution to address network connectivity also considers cost and network lifetime issues. Data latency issues are also considered when focusing on routing.

\section{Future research}

The future research of mobility management for mobile sensor nodes can be summarized by two main directions:

- We recommend studying the research of proximity performance in mobile sensor environments in an environment with obstacles. The goal is to ensure that mobile sensor nodes collaborate to simplify routing in such networks, especially because of the large issues that can arise when designing the appropriate routing protocols for such environments.

- It's necessary to pay attention to the event detection accuracy of mobile sensor nodes, and combine the event detection of the mobile node with the low-latency 
Table 1. Mobility Management Schemes Comparison

\begin{tabular}{|c|c|c|c|c|c|c|}
\hline Scheme & $\begin{array}{c}\text { Energy } \\
\text { efficiency }\end{array}$ & $\begin{array}{c}\text { Data } \\
\text { delay }\end{array}$ & Cost & Lifetime & $\begin{array}{c}\text { Simulation/ } \\
\text { Experiment }\end{array}$ & $\begin{array}{c}\text { Simulator } \\
\text { used }\end{array}$ \\
\hline RNC-RWP & Yes & Yes & No & No & Simulation & TOSSIM \\
\hline OACRPANM & Yes & No & Yes & No & Simulation & OMNeT ++ \\
\hline B-MAC & Yes & No & No & Yes & Simulation & - \\
\hline ResAll & Yes & No & No & No & Simulation & - \\
\hline FRAVD & Yes & Yes & No & No & Simulation & - \\
\hline
\end{tabular}

transmission of the static node, to implement the complete communication scheme in the MWSN.

\section{Conclusion}

In this paper, we study mobility management schemes for sensor nodes in the MWSN, and analyze these schemes mentioned in the literature. We observe that the mobility management schemes may have various properties and exhibit different mobility features. We believe that we can consider the more performance parameters in MWSN, or consider how mobile sensor nodes can perform well in the case of obstacles, and through the existing management schemes to improve the energy efficiency of mobile nodes in the future.

\section{Acknowledgment}

This research is supported by the National Natural Science Foundation of China under Grant Nos: 61562005; the Natural Science Foundation of Guangxi Province (Grant No. 2015GXNSFAA139286), and Thousands of Young and Middle-aged Backbone Teachers Training Program for Guangxi Higher Education (Education Department of Guangxi (2017) No.49).

\section{References}

1. Al-Muhtadi, Jalal, et al. "A Critical Analysis of Mobility Management Related Issues of Wireless Sensor Networks in Cyber Physical Systems." IEEE Access PP.99(2018):1-1.

2. Guezouli, Lyamine, et al. "Mobile sensor nodes collaboration to optimize routing process based mobility model." Wireless Networks and Mobile Communications (WINCOM), 2017 International Conference on. IEEE, (2017).

3. WU, Chun-hui, C. H. E. N. Bo, and Hong-sheng CHEN. "Obstacle-Avoiding Connectivity Restoration based on Node'S Movement and Deploying New Relay Node in Disjoint Mobile Sensor Networks." DEStech Transactions on Computer Science and Engineering cst (2017).

4. Khan, M. Umar, et al. "Various node mobility scenarios of wireless sensor networks based on BMAC protocol." HCT Information Technology Trends (ITT), 2017 Fourth. IEEE,( 2017).

5. Guo, Songtao, et al. "Energy efficiency maximization in mobile wireless energy harvesting sensor networks." IEEE Transactions on Mobile Computing 17.7 (2018): 1524-1537.

6. Huang, Mingfeng, et al. "A low-latency communication scheme for mobile wireless sensor control systems." IEEE Transactions on Systems, Man, and Cybernetics: Systems (2018).

7. Guo, Songtao, Chunrong He, and Yuanyuan Yang. "ResAll: Energy efficiency maximization for wireless energy harvesting sensor networks." Sensing, Communication, and Networking (SECON), 2015 12th Annual IEEE International Conference on. IEEE, (2015).

8. David, Bjohnson. "The dynamic source routing protocol for mobile ad hoc networks." Internet Draft, draft-ietf-manet-dsr-04. txt (2000).

9. Sir, Mustafa Y., et al. "An optimization-based approach for connecting partitioned mobile sensor/actuator networks." Computer Communications Workshops (INFOCOM WKSHPS), 2011 IEEE Conference on. IEEE, (2011)

10. Wu, Chun Hui, and Hong Sheng Chen. "Combining Power Adjustment and Node's Movement for Connectivity Restoration in Sensor Networks." Applied Mechanics and Materials. Vol. 644. Trans Tech Publications, (2014).

11. Thanigaivelu, K., and K. Murugan. "Impact of sink mobility on network performance in wireless sensor networks." 2009 First International Conference on Networks \& Communications. IEEE, (2009). 